\title{
Homopolyvalent antibody-antigen interaction kinetic studies with use of a dual-polarization interferometric biosensor
}

\author{
Shiming Lin ${ }^{\mathrm{a}, \mathrm{b}, *}$, Chih-Kung Lee ${ }^{\mathrm{b}}$, Yin-Hang Lin ${ }^{\mathrm{c}}$, Shih-Yuan Lee ${ }^{\mathrm{c}}$, \\ Bor-Ching Sheu ${ }^{\text {d }}$, Jui-Chang Tsai ${ }^{\text {a }}$, Su-Ming Hsu ${ }^{\mathrm{e}}$ \\ ${ }^{a}$ Center for Optoelectronic Biomedicine, National Taiwan University, 1-1 Jen-Ai Road, Taipei, Taiwan \\ ${ }^{\mathrm{b}}$ Institute of Applied Mechanics, National Taiwan University, Taipei, Taiwan \\ ${ }^{\mathrm{c}}$ Department of Chemistry, Tamkang University, Tamsui, Taiwan \\ ${ }^{\mathrm{d}}$ Department of Obstetrics and Gynecology, National Taiwan University, Taipei, Taiwan \\ ${ }^{\mathrm{e}}$ Institute of Pathology, National Taiwan University, Taipei, Taiwan
}

Received 23 October 2005; received in revised form 19 January 2006; accepted 17 February 2006

Available online 29 March 2006

\begin{abstract}
We used dual-polarization interferometry (DPI) to study the interaction kinetics between a 'homopolyvalent' antigen (Ag) and a monoclonal antibody (Ab). A model system, which uses a monoclonal Ab against a homopentameric Ag, C-reactive protein (CRP), is presented with principle and experiments for the study of the interactions between an $\mathrm{Ab}$ and an $\mathrm{Ag}$ that has multiple identical epitopes. This allows evaluation of the dissociation constant $\left(K_{\mathrm{D}}\right)$ and of the binding stoichiometry by DPI based on measurements of phase changes of Ab-Ag complexes in the transverse magnetic (TM) and transverse electric (TE) polarization modes. The average experimental value of $K_{\mathrm{D}}$ found by the DPI technique for anti-CRP Ab was shown to be in close agreement with the value obtained by an indirect competition-enzyme-linked immunosorbent assay (ELISA). Moreover, the total number of $\mathrm{Ab}$ combining sites on the DPI sensor chip was calculated, and the binding stoichiometry of the surface Ag-Ab complex was obtained. This study illustrates the advantages of the DPI method in biosensing in its capacity for simultaneous evaluation of the thickness and refractive index (density, mass) of adsorbed layers. This allowed a comprehensive analysis of affinity reactions between an Ab having two binding sites and a multi-sited Ag.
\end{abstract}

(C) 2006 Elsevier B.V. All rights reserved.

Keywords: Dual-polarization interferometry; C-reactive protein; Binding constant; Affinity; Stoichiometry; Homopolyvalence

\section{Introduction}

Based upon the law of mass action, several experimental approaches, for example, equilibrium dialysis (Eisen and Karush, 1949), fluorescence quenching and enhancement (Parker et al., 1967), and immunoaffinity capillary electrophoresis (Tseng et al., 2002) have been used for determination of the binding constant (affinity) for simple anti-(hapten)-hapten reactions. However, the determination of the affinity of an antiprotein antibody $(\mathrm{Ab})$ is complicated by the multivalence (multiple epitopes) of the antigen (Ag) (Friguet et al., 1985; Lin et al., 1997, 2000; Lin and Hsu, 1999). One Ab molecule, immunoglobulin $\mathrm{G}(\mathrm{IgG})$, has two identical combining sites and

\footnotetext{
* Corresponding author. Tel.: +886 23123456x8458; fax: +8862 23949125 . E-mail address: sml@ntumc.org (S. Lin).
}

can combine with two separate homologous hapten molecules, which have two identical antigenic determinants (epitopes, the regions of an $\mathrm{Ag}$ that bind to an $\mathrm{Ab}$ ) on one polymeric $\mathrm{Ag}$, or with separate epitopes on two Ag molecules (LeFor and Bauer, 1970).

A variety of experimental approaches have been developed which facilitate the verification of an interaction and the calculation of binding parameters such as the binding constant and stoichiometry for the interaction between a monovalent $\mathrm{Ab}$ and a homopolymer $\mathrm{Ag}$ that contains multiple copies of the same epitope (Berzofsky and Berkower, 1983; Rich and Myszka, 2003; Lin and Hsu, 2002, 2005). However, of the various existing methods for estimating the binding constant, most demand radioactive labeling or determination of the chromophoric characteristics of the interacting compounds, and these methods may require secondary reagents as well as an additional immobilization process for measurement at the liquid-solid interface. A 


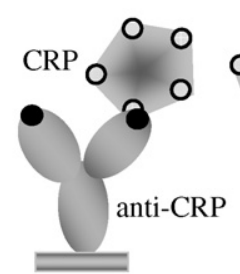

(A) $\mathrm{Ag}_{1} \mathrm{Ab}_{1}$,

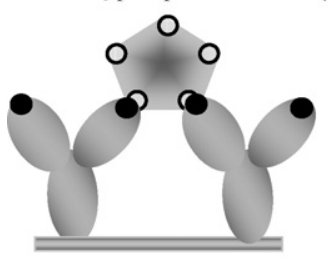

(C) $\mathrm{Ag}_{1} \mathrm{Ab}_{2}$

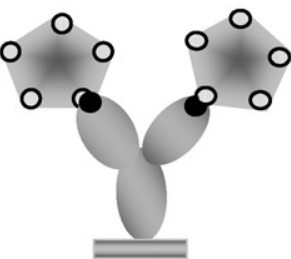

(B) $\mathrm{Ag}_{2} \mathrm{Ab}_{1}$

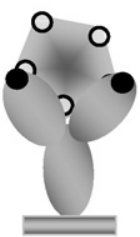

(D) $\mathrm{Ag}_{1} \mathrm{Ab}_{1}$,

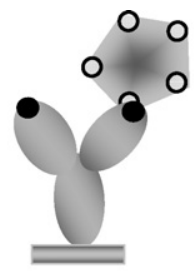

(1) in Ag excess

(2) conformational restraint of $\mathrm{Ag}$

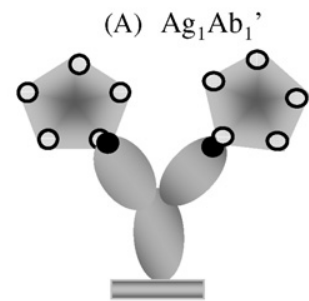

(B) $\mathrm{Ag}_{2} \mathrm{Ab}_{1}$

Fig. 1. Scheme of the binding of homopolyvalent CRP Ag(s) to divalent monoclonal anti-CRP Ab(s). (A) One Ab molecule binding to one epitope on an Ag to form an $\mathrm{Ag}_{1} \mathrm{Ab}_{1}^{\prime}$ complex. (B) One $\mathrm{Ab}$ molecule binding to separate epitopes on two $\mathrm{Ags}\left(\mathrm{Ag}_{2} \mathrm{Ab}_{1}\right.$ complex). (C) One $\mathrm{Ag}$ bound by two Abs on separate epitopes $\left(\mathrm{Ag}_{1} \mathrm{Ab}_{2}\right.$ complex). (D) One $\mathrm{Ab}$ molecule binding to two separate identical epitopes on an $\mathrm{Ag}$ to form an $\mathrm{Ag}_{1} \mathrm{Ab}_{1}^{\prime \prime}$ complex.

variety of biosensors have been developed in the last decade to examined binding interactions at sensor surfaces (Ramsay, 1998). Some of the more sensitive detection methods rely on evanescent wave techniques, e.g., surface plasmon resonance (SPR) phenomenon (Lin et al., 2005). Unfortunately, the physics of SPR techniques restricts measurements to single, transverse magnetic (TM) polarizations such that it is not possible to ascertain the absolute magnitude or nature of molecular events (Gestwicki et al., 2001; Cross et al., 2003). Dual-polarization interferometry (DPI), a new optical biosensor method developed recently, has been introduced for the study of bioaffinity interactions (Cross et al., 2003; Biehle et al., 2004) and the evaluation of changes in protein structure (Swann et al., 2004). By addressing the waveguide structure with alternate polarizations, one can determine the optogeometrical properties (density and thickness) of adsorbed protein layers at the sensor (solid)-liquid interface. Differences in the waveguide mode dispersion between the TM and transverse electric (TE) modes can allow unique solutions for surface concentrations of adsorbed proteins to be determined at all stages during the process of formation of protein layers (Cross et al., 2000, 2003, 2004; Worth et al., 2001). Efforts have been made to use DPI for observing biotin-streptavidin complex formation (Cross et al., 2003 ), analyzing lysozyme adsorption at various $\mathrm{pH}$ values ( $\mathrm{Lu}$ et al., 2004), detecting Apo E-specific interactions with tissue plasminogen activator (Biehle et al., 2004), measuring changes in streptavidin structure on binding of D-biotin (Swann et al., 2004), analyzing thin films of surfactants (Cross et al., 2004), and studying the interfacial adsorption of fibrinogen (Armstrong et al., 2004). However, the feasibility of studying the multivalent interaction kinetics of divalent Abs with homopolyvalent Ags by the DPI technique has not yet been investigated. The determination of the binding constant and stoichiometry of an $\mathrm{Ab}$ is complicated by the homopolyvalence (multiple identical epitopes) of the Ag.
C-reactive protein (CRP), an important risk factor for atherosclerosis and coronary heart disease (Tracy, 2003), has proved useful as prognostic indicator in acute coronary syndromes and in predicting future cardiovascular events in apparently healthy men and women (Blake et al., 2003). We report here on a new approach to determine the binding constant and stoichiometry for $\mathrm{Ab}-\mathrm{Ag}$ interactions by using unlabeled reactants at the liquid-solid (chip) interface. We focused on the use of a monoclonal anti-CRP Ab against a 'homopolyvalent' Ag, CRP, a homopentamer composed of five noncovalently associated protomers arranged symmetrically around a central pore (Motie et al., 1996), as a model system for evaluating the applicability of using DPI at an Ag excess for determination of the binding constant and stoichiometry based on the law of mass action for interactions between monovalent $\mathrm{Ab}$ and homopolyvalent $\mathrm{Ag}$ which contains multiple copies of the same epitope (as in the scheme illustrated in Fig. 1).

\section{Materials and methods}

\subsection{Amination of DPI sensor chip}

Measurements of surface layer thickness and density were made in real time with use of an AnaLight ${ }^{\circledR}$ Bio200 DPI biosensor (Farfield Sensors Ltd., Salford, UK). The functionalization of a DPI sensor chip (Farfield Sensors Ltd.) is based upon the method proposed by Weetall and Filbert (1974) and Weetall (1976). The bare sensor chip was first cleaned with a solution of $80 \%$ ethanol $/ 20 \%$ water for $5 \mathrm{~min}$ and then silanized with a $5 \%$ solution of 3-aminopropyltriethoxysilane (3-APTES) (Fluka Chemie, Buchs, Switzerland) in 5\% ethanol/95\% water at room temperature for $15 \mathrm{~min}$. The sensor chip was then rinsed with the 5\% ethanol/95\% water solution, followed by air drying for $15-30 \mathrm{~min}$. 


\subsection{Surface CRP-anti-CRP interactions}

A DPI sensor chip with amine functions covalently bound to the surface (Section 2.1) was mounted in an AnaLight ${ }^{\circledR}$ Bio200 DPI instrument (Farfield Sensors Ltd.). All protocols were carried out on the instrument at $22{ }^{\circ} \mathrm{C}$, with use of the standard fluidic system as supplied. PBS buffer $(\mathrm{pH}$ 7.4) flowed over the sensor chip at a rate of $50 \mu \mathrm{l} / \mathrm{min}$. Once the baseline sensor response had stabilized, an $80 \%$ ethanol $/ 20 \%$ water solution was made to flow over the sensor for $4 \mathrm{~min}$, followed by PBS buffer, allowing the baseline to stabilize once again. We injected distilled water for $4 \mathrm{~min}$ in order to determine the refractive index of the buffer solution, which was then used for performing the bulk index calibration. The flow was then reduced to $20 \mu \mathrm{l} / \mathrm{min}$, and 4\% glutaraldehyde solution (Riedel-deHaen, 62621, Berlin, Germany) in PBS buffer was injected and made to flow over the sensor chip for $5 \mathrm{~min}$ while maintaining the flow was maintained. The sensor chip was rinsed with PBS for 15 mins. Monoclonal anti-CRP IgG (100 $\mu \mathrm{g} / \mathrm{ml})$ (Sigma-Aldrich, C1688, Saint Louis, USA) in PBS buffer was then injected at a flow rate of $20 \mu \mathrm{l} / \mathrm{min}$ for $10 \mathrm{~min}$ while the flow was maintained before rinsing once again in PBS buffer for $10 \mathrm{~min}$. Unreacted aldehydes were deactivated by reaction with a large excess $(100 \mu \mathrm{l})$ of $1 \mathrm{M}$ ethanolamine. The baseline response was then allowed to stabilize for $10 \mathrm{~min}$ before $0.01 \mu \mathrm{g} / \mathrm{ml} \mathrm{CRP} \mathrm{(Sigma-Aldrich,} \mathrm{I5154,}$ Saint Louis, USA) was injected over the sensor chip and allowed to interact freely with the immobilized anti-CRP for $10 \mathrm{~min}$. We used the same anti-CRP surface repeatedly to study various concentrations (from 1 to $30 \mu \mathrm{g} / \mathrm{ml}$ ) of CRP samples. This was accomplished by washing away the associated CRP molecules with acidic buffer. Glycine buffer, 0.1 M (pH 2.5), was used for regenerating all surfaces bound by CRPs.

\subsection{Affinity measurements by an indirect ELISA}

An indirect competition ELISA experiment was performed according to the method developed by Friguet et al. (1985). The Ag (CRP) at various concentrations $(0.3-3 \mu \mathrm{M})$ was incubated in buffer solution with the monoclonal Ab (anti-CRP) at a constant concentration (13.3 nM) until equilibrium was reached. The binding constant in solution was determined by the equation (Friguet et al., 1985): $(1 / v)=1+K_{\mathrm{D}}\left(1 / a_{\mathrm{o}}\right) ; v$ is the fraction of bound $\mathrm{Ab}, a_{\mathrm{o}}$ the total concentration of $\mathrm{Ag}$, and $K_{\mathrm{D}}$ is the dissociation constant at equilibrium. $v$ corresponds to $\left(A_{\mathrm{O}}-A\right) / A_{\mathrm{O}}$, where $A$ is the absorbance measured in the ELISA at a given concentration and $A_{\mathrm{o}}$ is the absorbance measured for the $\mathrm{Ab}$ in the absence of $\mathrm{Ag}$. The $K_{\mathrm{D}}$ was then calculated from the equation of the best-fit straight line with slope $K_{\mathrm{D}}$.

\section{Theoretical considerations}

One can assume that the two combining sites of a divalent monoclonal $\mathrm{Ab}$, anti-CRP IgG, are equivalent and that neither is affected by events at the other. A divalent anti-CRP Ab can therefore be regarded as a monovalent molecule. The determination of the binding constant of an $\mathrm{Ab}$ is complicated by the homopolyvalence (presence of multiple identical epitopes) of the Ag, CRP; however, in Ag excess, the effect of the multiplicity of epitopes can be reduced (Fig. 1). Under these conditions, based on statistical considerations, the likelihood of a single $\mathrm{Ag}$ molecule binding to both $\mathrm{Ab}$ molecules (Fig. $1 \mathrm{C}, \mathrm{Ag}_{1} \mathrm{Ab}_{2}$ ) will be minimal. At a concentration of Ag near the dissociation constant, the presence of $1: 1$ complexes as $\mathrm{Ag}_{1} \mathrm{Ab}_{1}^{\prime}$ (Fig. 1A) or as a singly bonded species $\mathrm{Ag}_{1} \mathrm{Ab}_{1}^{\prime \prime}$ (Fig. 1D) cannot be neglected. However, some conformational restraint on the Ag may inhibit the divalent interaction in the complex $\mathrm{Ag}_{1} \mathrm{Ab}_{1}^{\prime \prime}$ (Fig. 1D), but the complex $\mathrm{Ag}_{1} \mathrm{Ab}_{1}^{\prime}$ (Fig. 1A) linked by a monovalent interaction should still exist; i.e., $\mathrm{Ag}_{1} \mathrm{Ab}_{1}^{\prime}$ (Fig. 1A) and $\mathrm{Ag}_{2} \mathrm{Ab}_{1}$ (Fig. 1B) complexes will predominate at high degrees of $\mathrm{Ag}$ excess and under the condition of conformational restraint on Ag (Mulligan et al., 1966; Singer and Campbell, 1952; LeFor and Bauer, 1970; Tseng et al., 2002).

The $\mathrm{Ag}-\mathrm{Ab}$ interaction in which the $\mathrm{Ab}$ (anti-CRP $\mathrm{IgG}$ ) is immobilized on a DPI sensor chip and the Ag (CRP) is transported by diffusion and flow to the sensor surface. The rate of the $\mathrm{Ag}-\mathrm{Ab}$ reaction at the solid-liquid interface to form an $\mathrm{AgAb}$ complex at time $t$ may be described as (Glaser, 1993):

$\frac{\mathrm{d} S_{\mathrm{Ag}}}{\mathrm{d} t}=k_{\mathrm{a}} C S-k_{\mathrm{d}} S_{\mathrm{Ag}}$,

where $C$ is concentration of $\mathrm{Ag}$ in solution at the surface $(\mathrm{M}), S_{\mathrm{Ag}}$ the surface concentration of $\mathrm{Ag}$ bound by the $\mathrm{Ab}\left(\mathrm{mol} / \mathrm{cm}^{2}\right), S$ the surface concentration of unoccupied Ab epitopes $\left(\mathrm{mol} / \mathrm{cm}^{2}\right)$, and $k_{\mathrm{a}}, k_{\mathrm{d}}$ are the association rate constant $\left(\mathrm{M}^{-1} \mathrm{~s}^{-1}\right)$ and dissociation rate constant $\left(\mathrm{s}^{-1}\right)$ for $\mathrm{Ag}$.

The concentration of unoccupied Ab epitopes, $S$, is the difference between the total amount of $\mathrm{Ab}$ epitopes on the sensor surface $\alpha S_{\mathrm{Ab}}$ and the amount of Ab combining sites blocked by $\operatorname{Ag} \beta S_{\mathrm{Ag}}$ :

$S=\alpha S_{\mathrm{Ab}}-\beta S_{\mathrm{Ag}}$,

where $S_{\mathrm{Ab}}$ is the surface concentration of the $\mathrm{Ab}\left(\mathrm{mol} / \mathrm{cm}^{2}\right), \alpha$ the number of exposed combining sites per $\mathrm{Ab}$ molecule or the number of $\mathrm{Ag}$ molecules that can bind to one $\mathrm{Ab}$ molecule, and $\beta$ is the number of blocked combining sites per bound $\mathrm{Ag}$.

Substituting in the rate equation (1) for formation of $\mathrm{AgAb}$ complex gives

$\frac{\mathrm{d} S_{\mathrm{Ag}}}{\mathrm{d} t}=k_{\mathrm{a}} C\left(\alpha S_{\mathrm{Ab}}-\beta S_{\mathrm{Ag}}\right)-k_{\mathrm{d}} S_{\mathrm{Ag}}$.

Data obtained at equilibrium, where $\left(\mathrm{d} S_{\mathrm{Ag}} / \mathrm{d} t\right)=0$, are influenced by the terms $\alpha$ and $\beta$. We obtain from Eq. (3):

$S_{\mathrm{Ag}}=\frac{\alpha S_{\mathrm{Ab}} C}{K_{\mathrm{D}}+\beta C}$,

where $K_{\mathrm{D}}=k_{\mathrm{d}} / k_{\mathrm{a}}\left(\mathrm{mol} / \mathrm{cm}^{3}=10^{3} \mathrm{M}\right)$ represents the dissociation constant.

If the total amount of Ag available for binding is much greater than the amount of $\mathrm{Ab}$, i.e., if $\mathrm{Ag}$ is present in excess, then $C \approx C_{\mathrm{t}}$, where $C_{\mathrm{t}}$ is the total Ag concentration loaded. Moreover, $\mathrm{Ag}_{1} \mathrm{Ab}_{1}^{\prime}$ and $\mathrm{Ag}_{2} \mathrm{Ab}_{1}$ complexes will predominate at high degrees of $\mathrm{Ab}$ excess and under the condition of conformational restraint of $\mathrm{Ag}$ (Fig. 1); i.e., if only one epitope is blocked per bound $\mathrm{Ag}$ either in $\mathrm{Ag}_{1} \mathrm{Ab}_{1}^{\prime \prime}$ or $\mathrm{Ab}_{2} \mathrm{Ag}_{1}$ complexes, then $\beta=1$. 
In such situations, Eq. (4) can be expressed as:

$\frac{1}{S_{\mathrm{Ag}}}=\frac{1}{\alpha S_{\mathrm{Ab}}}+\left(\frac{K_{\mathrm{D}}}{\alpha S_{\mathrm{Ab}}}\right)\left(\frac{1}{C_{\mathrm{t}}}\right)$.

Data analysis can be performed according to Eq. (5). The reciprocals of the surface concentration of $\mathrm{Ag}\left(1 / S_{\mathrm{Ag}}\right)$ and the concentration of total $\mathrm{Ag}\left(1 / C_{\mathrm{t}}\right)$ is plotted according to Eq. (5). In the linearized plot, the dissociation constant $K_{\mathrm{D}}$ may be determined from the intercept $(I)$ and slope $(S)$ of the best fit-straight line as the value of $(S / I)$. Moreover, the total number of Ab combining sites $\left(\alpha S_{\mathrm{Ab}}\right)$ on the sensor surface may be calculated as the value of $(1 / I)$.

\section{Results and discussion}

In a DPI sensor one of the reactants, i.e., anti-CRP, is immobilised on a dual-waveguide sensor chip surface. The other reactant, CRP, is transported by diffusion, and flows to the sensor surface, where it reacts with immobilised anti-CRP. Changes in the phases referred TM phase and TE phase are followed as a function of time and presented as a sensorgram (Fig. 2). The DPI sensor monitored the responses in TM and TE modes in all experimental steps, including: (1) the immobilization of anti-CRP IgG on the sensor chip (points a-b), (2) the deactivation of unreacted aldehyde sites of the aminated chip surface with an ethanolamine (points b-c), and (3) the interaction reactions of immobilised anti-CRP IgG with its Ag CRP at the solid (chip)-liquid interface (points c-d). Anti-CRP was immobilized on the aminated sensor chip by the formation of $-\mathrm{CH}=\mathrm{N}-$ bonding with the glutaldehyde. The covalent immobilization process improves the stability of anti-CRP molecules adsorbed on solid surfaces and permits the preparation of biologically active $\mathrm{Ab}$ interfaces for further $\mathrm{Ab}-\mathrm{Ag}$ interaction kinetic assay.

The refractive index and thickness data can be transformed into a surface coverage, provided we have reliable, independent data for the refractive index and density of the molecules

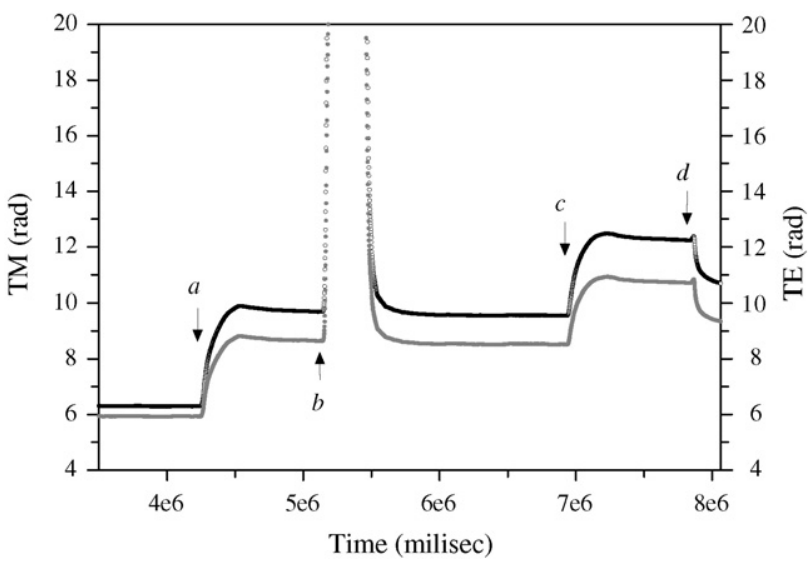

Fig. 2. Raw phase data for the immobilization of anti-CRP and the binding of CRP to a dual-waveguide sensor surface (black line denotes TM response and gray line denotes TE response). Point a shows addition of Ab (anti-CRP IgG) to the glutaldehyde-functionalized DPI sensor chip. Point $b$ shows the addition of ethanolamine to deactivate unreacted aldehyde sites. Point $\mathrm{c}$, the addition of $\mathrm{Ag}(\mathrm{CRP})$ to react with $\mathrm{Ab}$, and point d, the return to buffer wash. of interest. The surface concentration, $S_{\mathrm{Ab}}$, is obtained from $S_{\mathrm{Ab}}=(\rho / M) \times d$, where $\rho$ is the density of the molecular layer, $M$ the average molecular mass, and $d$ is the thickness of the deposited molecular layer. The density can be found from the measured refractive index of the Ab layer, $n$, under the assumption of additivity of the constituents, as follows: $\rho=\left(n-n_{\mathrm{W}}\right) /\left(n_{\mathrm{B}}-n_{\mathrm{W}}\right) \times \rho_{\mathrm{B}}$, where $n_{\mathrm{W}}$ and $n_{\mathrm{B}}$ are the indices of pure water and PBS buffer, respectively, and $\rho_{\mathrm{B}}$ is the density of the PBS buffer.

As shown in Fig. 3, the phase change (Fig. 3A) data in radians (rad) has been resolved into thickness and density changes
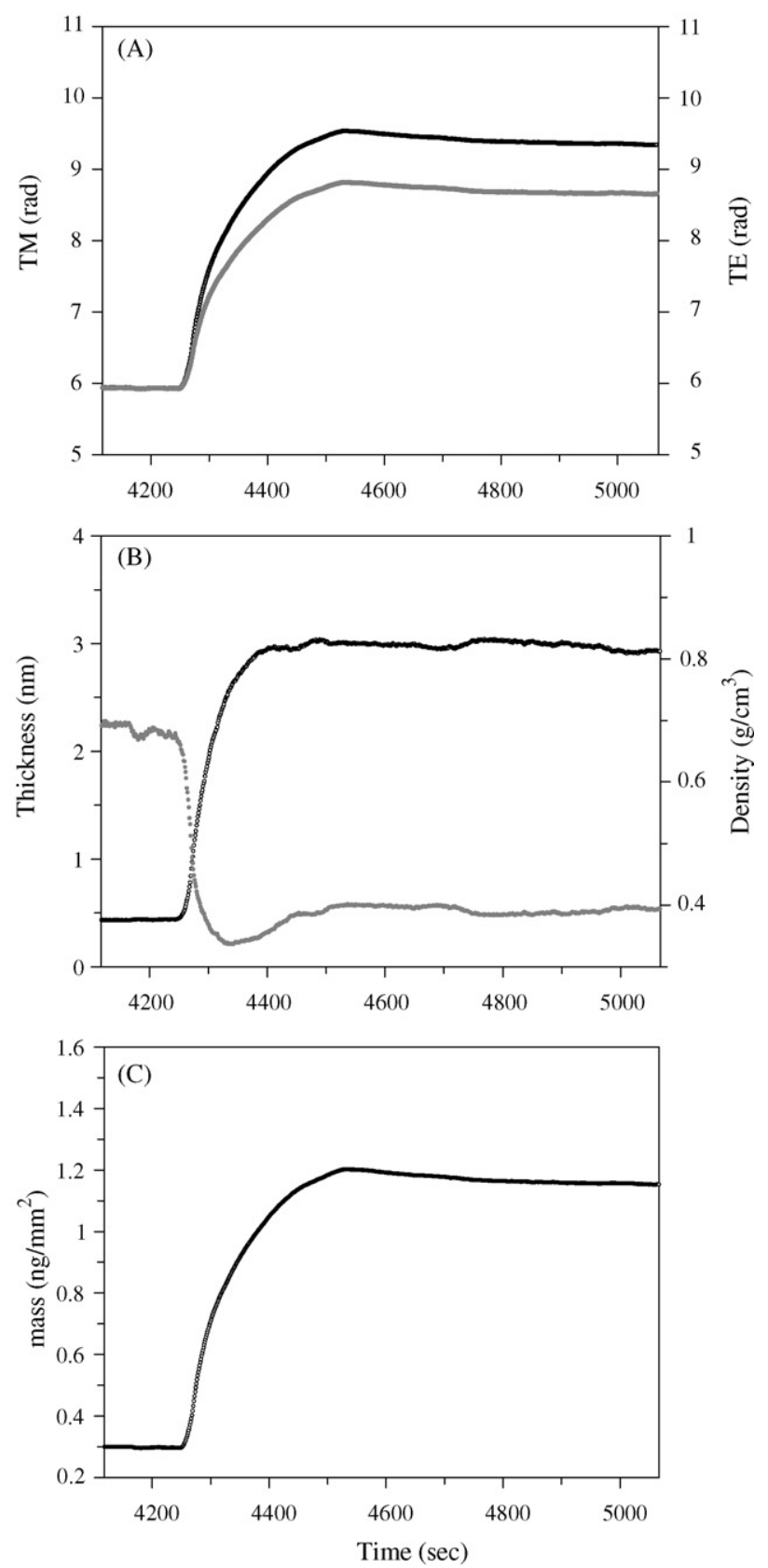

Fig. 3. Immobilization of anti-CRP on a glutaldehyde-functionalized sensor chip surface as shown by the measured TM (black) and TE (gray) phase changes (A) together with the resolved layer thickness (black line) and density (gray line) (B), and mass (surface concentration) (C). 
(Fig. 3B) for the anti-CRP adlayer by the DPI instrument (Cross et al., 2003). The thickness and density data can be transformed into a surface coverage (mass) (Fig. 3C). The surface concentration, $S_{\mathrm{Ab}}$, was obtained from $S_{\mathrm{Ab}}=(\rho / M) \times d$, where $\rho$ is the density of the IgG layer, $M$ is the average molecular mass of $\mathrm{IgG}(\sim 150 \mathrm{kDa})$, and $d$ is the thickness of the deposited IgG layer. The layer thickness after the immobilization of antiCRP IgG is observed to increase by $2.49 \mathrm{~nm}(20 \mu \mathrm{g} / \mathrm{ml} \mathrm{IgG}$ added). An IgG molecule is characterized by three discrete structural units, antigen binding is via two identical fragment antigen binding (Fab) fragments, whereas the effector functions are in the third fragment crystalline $(\mathrm{Fc})$ unit (Marquart and Deisenhofer, 1982). X-ray crystallography studies show IgG to be a Y-shaped molecule of overall dimensions approximately $14.2 \mathrm{~nm} \times 8.5 \mathrm{~nm} \times 4.0 \mathrm{~nm}$; the Fc tail is $\sim 7.2 \mathrm{~nm}$ long and has a cross section of about $4.5 \mathrm{~nm} \times 3.8 \mathrm{~nm}$, whereas the two Fab arms are $\sim 6 \mathrm{~nm}$ long and about $4.0 \mathrm{~nm} \times 4.0 \mathrm{~nm}$ in cross section (Ahluwalia et al., 1992). Therefore, the thickness increase $(2.49 \mathrm{~nm})$ observed is attributed to the formation of a 'partial' monolayer of protein immobilized randomly on the sensor surface. On the other hand, from the mass loading (surface concentration), it is possible to calculate the area per molecule of surface coverage, which provides an additional indication of the likelihood that a monolayer or a multilayer has been formed. When the measured area per molecule is equal to the theoretical saturated area per molecule, a monolayer structure would be expected, and when the area per molecule is significantly lower than the theoretical saturated area per molecule, the structure is likely to be multilayer in nature. The 'theoretical' saturated monolayer area per IgG molecule is estimated to be about $158.4 \mathrm{~nm}^{2}$ (the approximate diameter of the IgG molecule is about $14.2 \mathrm{~nm}$ (Ahluwalia et al., 1992) and is assumed to have a circular cross-section). Taking the example (Fig. 3C), a surface coverage of $\mathrm{IgG}$ of $5.72 \times 10^{-15} \mathrm{~mol} / \mathrm{mm}^{2}$ is observed, which is equivalent to an area per $\mathrm{IgG}$ molecule of $291 \mathrm{~nm}^{2}$. Given that this figure $\left(291 \mathrm{~nm}^{2} /\right.$ molecule $)$ is greater than the 'theoretical' saturated area per molecule ( $158.4 \mathrm{~nm}^{2} /$ molecule), the most likely surface structure is that of a 'partial' monolayer coverage.

To prepare a full $\mathrm{Ab}$ monolayer for further determination of the binding constant for $\mathrm{Ag}-\mathrm{Ab}$ interaction kinetics, we varied the concentration of anti-CRP added to the glutaldehyde-functionalised sensor surface. Fig. 4A shows the resolved layer thickness and refractive index versus the concentration of anti-CRP added to the sensor surface. Examining the data obtained, one can see that, as the anti-CRP concentration increases, the adsorbed layer thickness increases and the refractive index (proportional to density) decreases. This suggests that the antibody molecules adopt a prone attitude at low concentrations, resulting in a thin, relatively dense layer coverage, which becomes less dense and thicker as the anti-CRP is approached and the antibody molecules adopt a more upright stance. Moreover, the thickness and density data can be transformed into surface coverage (Fig. 4B). In Fig. 4B, the surface concentration $S_{\mathrm{AB}}$ increases up to a maximum binding capacity (approximately $9.47 \times 10^{-15} \mathrm{~mol} / \mathrm{mm}^{2}$ ) and then levels off. This behaviour conforms to the standard Langmuir isotherm characteristics. Taking the example, a surface coverage of IgG
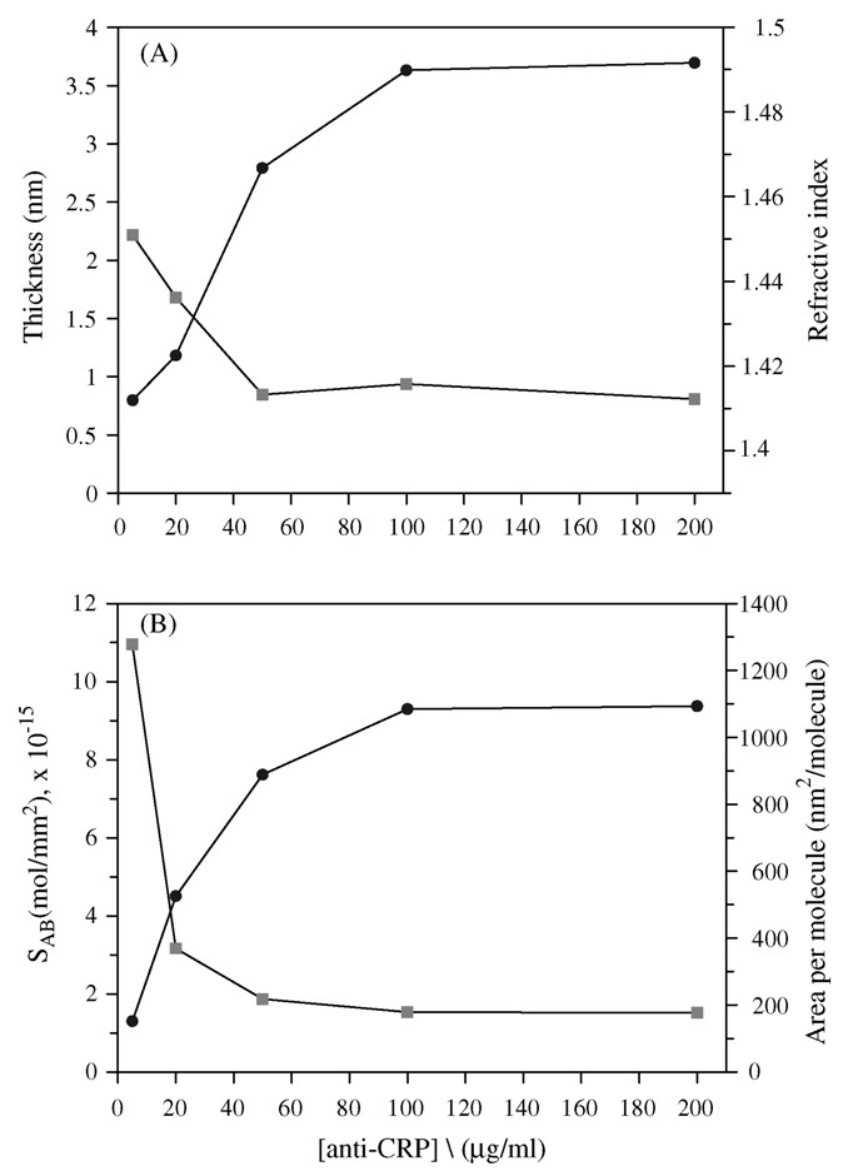

Fig. 4. Addition of various concentrations of monoclonal anti-CRP IgG onto a glutaldehyde-functionalized DPI sensor chip as shown by the resolved layer thickness (black line) and refractive index (gray line) (A) together with the transformed surface concentration $S_{\mathrm{AB}}$ of the IgG layer (black line) and the area per IgG molecule (gray line) (B). In (B), the surface concentration $S_{\mathrm{AB}}$ increases up to a maximum binding capacity (approximately $9.37 \times 10^{-15} \mathrm{mols} / \mathrm{mm}^{2}$ ) and then levels off.

of $9.47 \times 10^{-15} \mathrm{~mol} / \mathrm{mm}^{2}$ is observed, which is equivalent to an area per $\operatorname{IgG}$ molecule of $175.4 \mathrm{~nm}^{2}$. Given that this figure $\left(175.4 \mathrm{~nm}^{2} /\right.$ molecule $)$ is close to the 'theoretical' saturated area per molecule $\left(158.4 \mathrm{~nm}^{2} / \mathrm{molecule}\right)$, the most likely surface structure is that of a monolayer. Such a preparation of a monolayer $\mathrm{Ab}$ surface was then employed in the determination of the binding constant for $\mathrm{Ag}-\mathrm{Ab}$ interactions as described below.

Depending on the fixed surface concentration of $\mathrm{Ab}$ $\left(9.47 \times 10^{-15} \mathrm{~mol} / \mathrm{mm}^{2}\right.$ ), various concentrations (from 0.2 to $40 \mu \mathrm{g} / \mathrm{ml}$ ) of CRP samples were added to the Ab monolayer, and the TM and TE phase status of the Ab-Ag complex and the change in thickness and density will result in measurable mass changes (Fig. 5A), and the binding curve forms the basis for subsequent estimation of the binding constant $K_{\mathrm{D}}$ from the equation: $\left(1 / S_{\mathrm{Ag}}\right)=\left(1 / \alpha S_{\mathrm{Ab}}\right)+\left(K_{\mathrm{D}} / \alpha S_{\mathrm{Ab}}\right) \times\left(1 / C_{\mathrm{t}}\right)($ Eq. $(5))$. When a 1-20-fold concentration of $\mathrm{Ag}$ over $\mathrm{Ab}$ binding sites $(\sim 0.2 \mu \mathrm{g} / \mathrm{ml})$ was conducted with this $\mathrm{Ab}$, the observed plot was not linear from 0.2 to $4 \mu \mathrm{g} / \mathrm{ml}$ CRP. However, at a large Ag excess (25-200-fold molar excess), a linear plot of Eq. (5) was obtained from 5 to $40 \mu \mathrm{g} / \mathrm{ml}$ CRP for the anti-CRP Ab, as displayed in Fig. 5B. In the linearized plot, the $K_{\mathrm{D}}$ was determined 

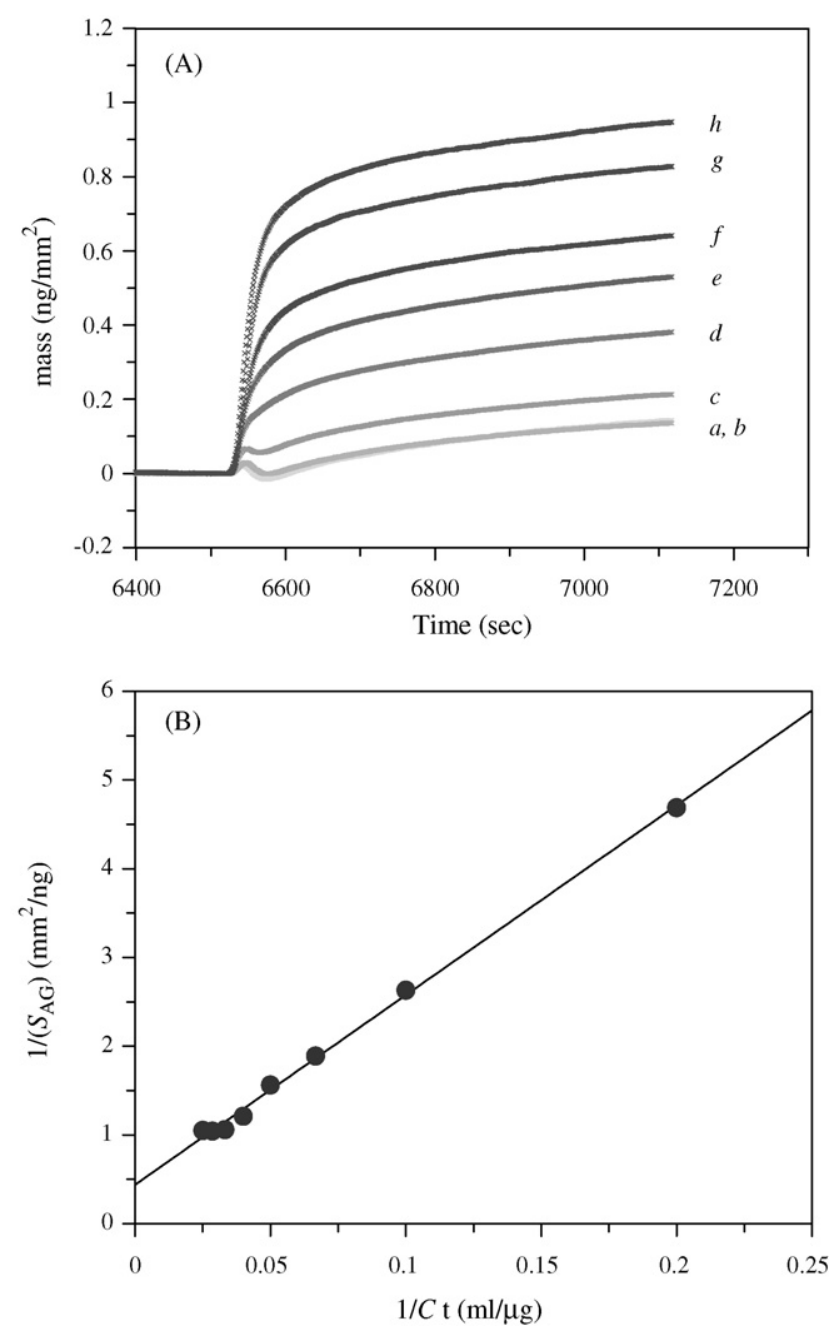

Fig. 5. (A) Binding curves of CRP to anti-CRP Ab at the silica/water interface by DPI. Various concentrations (a for $5 \mu \mathrm{g} / \mathrm{ml}$, b for $10 \mu \mathrm{g} / \mathrm{ml}$, c for $15 \mu \mathrm{g} / \mathrm{ml}$, d for $20 \mu \mathrm{g} / \mathrm{ml}$, e for $25 \mu \mathrm{g} / \mathrm{ml}$, f for $30 \mu \mathrm{g} / \mathrm{ml}$, g for $35 \mu \mathrm{g} / \mathrm{ml}$, and $\mathrm{h}$ for $40 \mu \mathrm{g} / \mathrm{ml}$ ) of CRP were added to a dual-waveguide sensor chip immobilized with anti-CRP Ab. (B) Determination of dissociation constant and stoichiometry for the interaction between anti-CRP Ab and homopolyvalent CRP. The dissociation constant $\left(K_{\mathrm{D}}=4.89 \times 10^{-7} \mathrm{M}\right)$, the total number of $\mathrm{Ab}$ combining sites $\left(\alpha S_{\mathrm{Ab}}=2.27 \mathrm{ng} / \mathrm{mm}^{2}\right)$, and the binding stoichiometry $(\alpha=1.61)$ were calculated from Eq. (5), for the best-fit straight line by linear regression (correlation coefficient $r=0.99$ ). In three determinations an experimental $K_{\mathrm{D}}$ of $(4.47 \pm 0.42) \times 10^{-7} \mathrm{M}$ and a stoichiometry of $1.56 \pm 0.06$ were obtained.

from the intercept $\left(I=0.44 \mathrm{~mm}^{2} / \mathrm{ng}\right)$ and slope $(S=21.38)$ of the best-fit straight line (correlation coefficient $r=0.99$ ) as the value of $(S / I)$ and found to be $4.89 \times 10^{-7} \mathrm{M}$. In three determinations, an dissociation constant $K_{\mathrm{D}}$ of $(4.47 \pm 0.32) \times 10^{-7} \mathrm{M}$ was obtained. Moreover, the total number of $\mathrm{Ab}$ combining sites $\left(\alpha S_{\mathrm{Ab}}=2.27 \mathrm{ng} / \mathrm{mm}^{2}\right)$ on the sensor surface was calculated as the value of $(1 / I)$. Furthermore, $\alpha$ the number of Ag molecules that can bind to one $\mathrm{Ab}$ molecule, was calculated from the surface concentration $S_{\mathrm{AB}}\left(1.41 \mathrm{ng} / \mathrm{mm}^{2}\right)$ and found to be 1.61 $\left(\alpha S_{\mathrm{Ab}} / S_{\mathrm{Ab}}=2.27 / 1.41\right)$.

In three determinations, an average $\alpha$ value, i.e., a average stoichiometry of $1.56 \pm 0.06$, was obtained. Assuming binding of CRP to the bivalent antibody ( $\mathrm{IgG}$ ), an estimated maximum of $78 \%$ of the anti-CRP IgG molecules retained the conformation

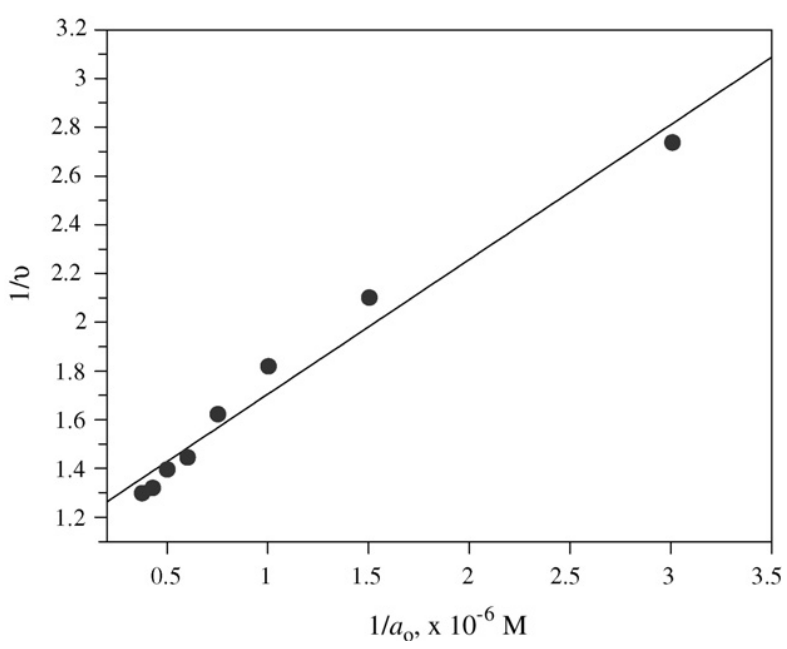

Fig. 6. Scatchard plot of CRP binding to monoclonal anti-CRP Ab measured by an indirect competition ELISA. The $K_{\mathrm{D}}$ was calculated from the equation, $(1 / v)=1+K_{\mathrm{D}}\left(1 / a_{\mathrm{o}}\right)$, of the best-fit straight line (correlation coefficient $r=0.98)$. Each experiment was repeated and samples were analyzed in duplicate. The experimental dissociation constant $K_{\mathrm{D}}$ value obtained is $(5.53 \pm 0.48) \times 10^{-7} \mathrm{M}$.

required to bind CRP. This observation can be attributed to the fact that the immobilization chemistry used involves glutaldehyde activation, which couples randomly through primary amino groups on the surface of IgG. Thus, multiple orientations of the $\mathrm{IgG}$ on the silicon chip surface are possible, depending on which primary amino groups bind to the glutaldehyde-functionalized silicon chip. One monoclonal anti-CRP IgG molecule has two identical combining sites and can be bound to two separate CRP molecules, and therefore the molar binding ratio (or $\alpha$ value) should ideally be 2.0. However, some orientations of the antiCRP IgG molecules on the sensor chip may result in an active IgG (about 78\%) that physically can bind the CRP in solution, whereas other orientations may result in an inactive $\mathrm{IgG}$ (about $22 \%$ ) because of potential bonding at or near the critical binding regions, thereby blocking these sites and leading to partial loss of binding activity.

To be able to validate the results obtained with this new DPI approach, we have measured the dissociation constant of the $\mathrm{Ab}$ (anti-CRP IgG)-Ag (CRP) complexes by an indirect competition ELISA method (Friguet et al., 1985), which can provide the real thermodynamic affinity of the $\mathrm{Ab}$ for its $\mathrm{Ag}$. As shown in Fig. 6, a linear fit (correlation coefficient $r=0.98$ ) was obtained. From linear regression, the experimental $K_{\mathrm{D}}$ value obtained is $(5.53 \pm 0.48) \times 10^{-7} \mathrm{M}$. The experimental value of $K_{\mathrm{D}}\left((4.47 \pm 0.32) \times 10^{-7} \mathrm{M}\right)$ calculated by a DPI method for anti-CRP mAb was shown to be in close agreement with that $\left((5.53 \pm 0.48) \times 10^{-7} \mathrm{M}\right)$ obtained by the indirect ELISA procedure.

\section{Conclusions}

The interaction between a protein, $\mathrm{Ag}$, and the corresponding $\mathrm{Ab}$ is complex because of the heterogeneity and multiplicity of antigenic determinants. However, as shown by our studies in which we used an anti-CRP mAb bound to a homopolyvalent 
pentameric CRP Ag, approximations can be made which yield the linearization of Eq. (5), deduced under certain conditions where the total Ag concentration is in large excess over the total $\mathrm{Ab}$ concentration, and where the likelihood of both identical combining sites of an IgG binding to a single Ag molecule can be neglected due to conformational restraint on the Ag. When these requirements are fulfilled, the $K_{\mathrm{D}}$ value can be evaluated from the Eq. (5) by DPI based upon the law of mass action in monovalent mode. This is found to be in close agreement with the value obtained by an indirect competition method based on ELISA.

In addition, this DPI method also offers several advantages over those commonly used for measurement of Ab affinity. First, it provides quantitative information to a particularly high resolution in terms of thickness and density of thin $\mathrm{Ab}$ and $\mathrm{Ag}$ films in real time, which may be compared with other analytical techniques such as SPR, X-ray crystallography and neutron reflection (Cross et al., 2003). Moreover, it requires no labeling of either the $\mathrm{Ag}$ or the $\mathrm{Ab}$. Furthermore, compared to other optical biosensor, e.g., SPR or resonance mirror (RM) biosensor, DPI is a highly sensitive technique for measuring small-molecule interactions with protein molecules. It allows a quantitative assessment of the amount of the small-molecule $\mathrm{Ag}$ that binds to the $\mathrm{Ab}$ immobilized on the sensor surface. In view of the theoretical reliability and the sensitivity and rapidity of this DIP method (Cross et al., 2000, 2003, 2004; Sarkisov et al., 2001; Worth et al., 2001), when certain requirements are fulfilled, the method is likely to be of interest for studies of the interaction kinetics of monoclonal antibodies specific for any multivalent polymeric protein antigens. The more complicated interaction kinetics of polyclonal antibodies by DPI requires other considerations.

\section{References}

Ahluwalia, A., Rossi, D.D., Schirone, A., 1992. Thin Solid Films 210 (2), 726-729.

Armstrong, J., Salacinski, H.J., Mu, Q., Seifalian, A.M., Peel, L., Freeman, N., Holt, C.M., Lu, J.R., 2004. J. Phys.: Condens. Mater. 16, S2483S2491.
Berzofsky, J.A., Berkower, I.J., 1983. In: Paul, W.E. (Ed.), Antibody-Antigen Interaction. CRC Press, Boca Raton, pp. 595-644.

Biehle, S.J., Carrozzella, J., Shukla, R., Popplewell, J., Swann, M., Freeman, N., Clark, J.F., 2004. Biochim. Biophys. Acta 1689 (3), 244 251

Blake, G.J., Rifai, N., Buring, J.E., Ridker, P.M., 2003. Circulation 108 (24), 2993-2999.

Cross, G.H., Ren, Y., Swann, M.J., 2000. Analyst 125 (12), 21732175.

Cross, G.H., Reeves, A.A., Brand, S., Popplewell, J.F., Peel, L.L., Swann, M.J., Freeman, N.J., 2003. Biosens. Bioelectron. 19 (4), 383-390.

Cross, G.H., Reeves, A.A., Brand, S., Swann, M.J., Peel, L.L., Freeman, N.J., Lu, J.R., 2004. J. Phys. D: Appl. Phys. 37 (1), 74-80.

Eisen, H.N., Karush, F., 1949. J. Am. Chem. Soc. 71 (1), 363-369.

Friguet, B., Chaffotte, A.F., Djavadi-Ohaniance, L., Goldberg, M.E., 1985. J. Chromatogr. 77, 305-319.

Gestwicki, J.E., Hsieh, H.V., Pitner, J.B., 2001. Anal. Chem. 73, 5732-5737.

Glaser, R.W., 1993. Anal. Biochem. 213 (1), 152-161.

LeFor, W.M., Bauer, D.C., 1970. J. Immunol. 104 (5), 1276-1282.

Lin, S., Hsiao, I.Y., Hsu, S.M., 1997. Anal. Biochem. 254, 9-17.

Lin, S., Hsu, S.M., 1999. Electrophoresis 20, 3388-3395.

Lin, S., Tsai, J.C., Hsu, S.M., 2000. Anal. Biochem. 284, 422-426.

Lin, S., Hsu, S.M., 2002. Electrophoresis 23, 836-846.

Lin, S., Hsu, S.M., 2005. Anal. Biochem. 341, 1-15.

Lin, L.P., Huang, L.S., Lee, C.K., Lin, S., 2005. Curr. Drug Targets 5, 61-72.

Lu, J.R., Swann, M.J., Peel, L.L., Freeman, N.J., 2004. Langmuir 20 (5), $1827-1832$

Marquart, M., Deisenhofer, J., 1982. Immunol. Today 3 (6), 160-166.

Motie, M., Brockmeier, S., Potempa, L.A., 1996. J. Immunol. 156 (11), 4435-4441.

Mulligan, J.J., Osler, A.C., Rodriguez, E., 1966. J. Immunol. 96, 324-329.

Parker, C.W., Yoo, T.J., Johnson, M.C., Godt, S.M., 1967. Biochemistry 6 (11), 3408-3412.

Ramsay, G. (Ed.), 1998. Commercial Biosensor. Wiley Interscience.

Rich, R.L., Myszka, D.G., 2003. Trends Microbiol. 11 (3), 124-133.

Sarkisov, S.S., Diggs, D.E., Adamovsky, G., Curley, M.J., 2001. Appl. Opt. 40 (3), 349-359.

Singer, S.J., Campbell, D.H., 1952. J. Am. Chem. Soc. 74, 1974-1979.

Swann, M.J., Peel, L.L., Carrington, S., Freeman, N.J., 2004. Anal. Biochem. 329 (2), 190-198

Tracy, R.P., 2003. Int. J. Obes. 27, S29-S34.

Tseng, W.L., Chang, H.T., Hsu, S.M., Chen, R.J., Lin, S., 2002. Electrophoresis 23 (6), 836-846.

Weetall, H.H., Filbert, A.M., 1974. Methods Enzymol. 34, 59-72.

Weetall, H.H., 1976. Methods Enzymol. 44, 134-148.

Worth, C., Goldberg, B.B., Ruane, M., Ünlü, M.S., 2001. IEEE J. Sel. Top. Quantum. Electron. 7 (6), 874-877. 